\title{
Determining Potentials of Zero Charge of Metal Electrodes versus the Standard Hydrogen Electrode from Density-Functional-Theory-Based Molecular Dynamics
}

\author{
Jiabo Le, ${ }^{1,2}$ Marcella Iannuzzi, ${ }^{3}$ Angel Cuesta, ${ }^{2}$ and Jun Cheng ${ }^{1,2, *}$ \\ ${ }^{1}$ State Key Laboratory of Physical Chemistry of Solid Surfaces, iChEM, College of Chemistry and Chemical Engineering, \\ Xiamen University, Xiamen 361005, China \\ ${ }^{2}$ Department of Chemistry, University of Aberdeen, Aberdeen AB24 3UE, United Kingdom \\ ${ }^{3}$ Department of Physical Chemistry, University of Zürich, Winterthurerstrasse 190, 8057 Zürich, Switzerland
}

(Received 18 October 2016; published 5 July 2017)

\begin{abstract}
We develop a computationally efficient scheme to determine the potentials of zero charge (PZC) of metal-water interfaces with respect to the standard hydrogen electrode. We calculate the $\mathrm{PZC}$ of $\mathrm{Pt}(111)$, $\mathrm{Au}(111), \operatorname{Pd}(111)$ and $\operatorname{Ag}(111)$ at a good accuracy using this scheme. Moreover, we find that the interface dipole potentials are almost entirely caused by charge transfer from water to the surfaces, the magnitude of which depends on the bonding strength between water and the metals, while water orientation hardly contributes at the PZC conditions.
\end{abstract}

DOI: 10.1103/PhysRevLett.119.016801

Metal-water interfaces are of great technological importance in many energy storage and conversion devices such as fuel cells and batteries. Fundamentally, they are the primary subjects for studying electrochemical processes (i.e., electrocatalysis and corrosion) in electrochemistry and play a crucial role in the development of electric double layer (EDL) theories (i.e., Gouy-Chapman-Stern model). Direct probing of structures and dynamics of the interfaces at a molecular level is extremely challenging for experiment. First principles simulations, on the other hand, can offer detailed microscopic information on the interfaces. However, due to high computational costs, it was not long ago that $a b$ initio modeling of metal-water interfaces became affordable [1,2].

Potential of zero charge (PZC) is a fundamental concept in the EDL theories, defined as the potential at which no excess charge exists on metal surfaces, and deviation from the PZC will lead to attraction of counterions to the surfaces, building up the EDL [3]. Because of its significance to the microscopic understanding of an EDL and interfacial potentials, numerous experimental techniques have been developed to determine the PZC of metal electrodes, e.g., surface tension methods, capacitance measurement methods, CO charge-displacement methods, etc [4]. Despite repeated efforts, many measurements are still subject to uncertainties because of difficulties in preparing single crystal electrodes and excluding specific adsorption of electrolyte ions [5-7]. In the presence of specific adsorption, electrochemists distinguish the subtlety between the potential of zero total charge (PZTC) and the potential of zero free charge (PZFC), and only the latter is an intrinsic property of metal electrodes [8].

First principles calculation of well-defined metal surfaces is ideal for determining the PZFC. There are two issues in computational methods. First, how is the solvent treated in the simulation models? In the literature, water has often been treated with either an implicit dielectric continuum [9-11] or some representations of static water structures for efficiency [12]. It, however, has been reported that the dynamics of water on surfaces has significant effects on interface potentials [13]. As yet, very few studies have modeled full metal-water interfaces and accounted for water dynamics using density functional theory based molecular dynamics (DFTMD) $[14,15]$. Second, how are the computed potentials referenced to? The majority of previous calculations use a work function method in which a water-vapor interface has to be explicitly modeled and the vacuum potential is chosen as reference. This method can be dated back to the late 1980s for first principles calculations of band lineups at semiconductor heterojunctions using bulk electrostatic (Hartree) potentials as reference [16]. In electrochemical applications, the Hartree potential of bulk water requires appropriate statistical averaging. To compare with experiment, an extra step is needed to convert the computed "absolute" potentials into the standard hydrogen electrode (SHE) scale by subtracting the experimental value of the absolute SHE potential. However, a water-vapor interface is not as straightforward to model as it appears [17], and the experimental estimate of the SHE potential has an uncertainty on the order of $\sim 0.5 \mathrm{~V}(4.2-4.7 \mathrm{~V})$ [18].

Alternatively, a computational SHE method developed by Cheng and Sprik $[19,20]$ avoids treating the water-vapor interface and references the computed potentials to the SHE without the need for any experimental input, therefore allowing for direct comparison with experimental values. This method uses the solvation free energy of the aqueous proton $\mathrm{H}^{+}(\mathrm{aq})$ as reference, which is calculated using thermodynamic integration over a set of ensembles sampled by DFTMD [21,22]. This reference method has been validated at the various levels of electronic structure 
theories from the generalized gradient approximation (GGA), hybrid functionals to the random phase approximation (RPA) [23]. It has been used to calculate aqueous solutions [22] and a few semiconductor-water interfaces [24]. The obvious downside is the high computing costs that limit its wide applications to other complex systems including metal-water interfaces. In this Letter, we report the latest development of the computational SHE method that avoids expensive calculation of the solvation free energy of the proton in metal-water interface models, enabling us to calculate the PZC vs SHE of metal electrodes at an affordable cost. Four well documented transition metals showing diverse electrochemical properties, namely, $\operatorname{Pt}(111)$, $\operatorname{Pd}(111), \operatorname{Ag}(111)$ and $\mathrm{Au}(111)$, have been chosen to test the method.

The other main objective is to investigate the hydration effect on the band positions of transition metal surfaces, i.e., the PZC vs work function $(\Phi)$. This issue is related to the band alignment and formation of the Schottky barrier height (SBH) at metal-semiconductor heterojunctions. The often observed independence of the SBH on metal work functions is attributable to the pinning at gap states formed at interfacial regions [25]. In contrast, electrochemical experiments show a linear correlation between the PZC and $\Phi$ for main group $s p$ metals with a small constant shift due to interface effects [3]. Recent computational studies on semiconductor-water interfaces also indicate the complexity of interface dipole effects [19,26-28]. For example, using an implicit solvation model, Zunger et al. found a rather universal shift of $\sim 0.5 \mathrm{eV}$ in the band edges, irrespective of the material [26]. Sprik et al., however, showed a significant shift of $\sim 1.5 \mathrm{eV}$ at the $\mathrm{TiO}_{2}$-water interface using DFTMD [19]. A similar shift was also observed by Galli et al. on the hydrophilic $\mathrm{COOH}-$ terminated $\mathrm{Si}(111)$ surface [27]. In this work, we show that the interface dipole potentials caused by charge redistribution dominate the differences between the PZC and $\Phi$ on transition metals, the magnitude of which depends on the strength of chemical bonding between water and surfaces, while the water orientation at interface regions only shows a minor effect.

The computational SHE method has been derived in detail in previous publications [20-22,29], and briefly summarized in the Supplemental Material [30]. Here we simply write down the formula for the calculation of electronic energies vs the $\operatorname{SHE}\left(U^{\circ}\right)$ as follows:

$$
e U^{\circ}=\varepsilon^{(w)}+\Delta_{\mathrm{DP}} A_{\mathrm{H}_{3} \mathrm{O}^{+}}^{(w)}-\mu_{\mathrm{H}^{+}}^{g, \circ}-\Delta E_{\mathrm{ZP}},
$$

in which $\varepsilon^{(w)}$ stands for electronic ionization energies (vertical or adiabatic), $\Delta_{\mathrm{DP}} A_{\mathrm{H}_{3} \mathrm{O}^{+}}^{(w)}$ is the deprotonation free energy of $\mathrm{H}_{3} \mathrm{O}^{+}(\mathrm{aq})$ (i.e., equivalent of minus solvation free energy of a proton), $\mu_{\mathrm{H}^{+}}^{g, \circ}$ is the standard chemical potential of gas phase proton and $\Delta E_{\mathrm{ZP}}$ is a correction for the zero-point energy of the $\mathrm{O}-\mathrm{H}$ bond in $\mathrm{H}_{3} \mathrm{O}^{+}(\mathrm{aq})$. The latter two are known constants, $15.81 \mathrm{eV}$ and $0.35 \mathrm{eV}$, respectively $[21,29]$. Note that computed in periodically repeated simulation boxes, neither $\varepsilon^{(w)}$ nor $\Delta_{\mathrm{DP}} A_{\mathrm{H}_{3} \mathrm{O}^{+}}^{(w)}$ has physical meaning alone due to the artificial offset [Hartree potential shift (HPS)] in the potential reference under periodic boundary conditions (PBCs) [21]. When combining these two, the offsets in both terms cancel [see Fig. 1(a)]. This only works when both terms are computed in the same periodic models because the HPS is dependent on the composition and pseudopotentials of simulation cells, and the HPS varies with different model systems. To emphasize this fact, we explicitly add (w) as a superscript in $\varepsilon^{(w)}$ and $\Delta_{\mathrm{DP}} A_{\mathrm{H}_{3} \mathrm{O}^{+}}^{(w)}$ to indicate both energies need to be computed in the same box of liquid water. $\Delta_{\mathrm{DP}} A_{\mathrm{H}_{3} \mathrm{O}^{+}}^{(w)}$ has been calculated within an accuracy of $\sim 0.1 \mathrm{eV}$ using both 32 and 64 water boxes at various levels of electronic structure theories, and the recommended value is $15.35 \mathrm{eV}[22,23]$.

Turning to metal-water interfaces, we can similarly express the PZC vs SHE $\left(U_{\mathrm{PZC}}^{\circ}\right)$ as,

(a)
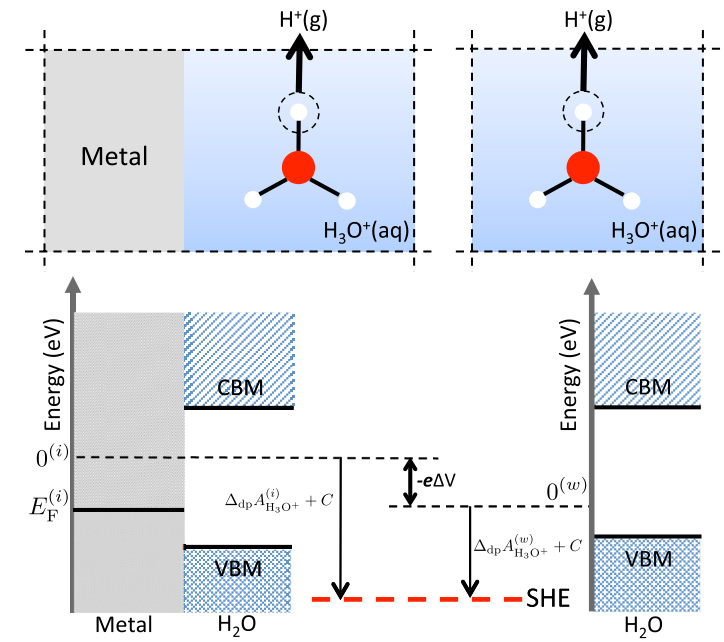

(b)



FIG. 1. (a) The upper panel sketches the process of computing the reference deprotonation free energies of a solvated hydronium ion in interface and pure water models under the $\operatorname{PBC}\left(\Delta_{\mathrm{DP}} A_{\mathrm{H}_{3} \mathrm{O}^{+}}^{(i)}\right.$ and $\Delta_{\mathrm{DP}} A_{\mathrm{H}_{3} \mathrm{O}^{+}}^{(w)}$, respectively). The lower panel indicates how the potential "zero" is aligned to the SHE scale. $C$ is a constant, equal to $-\mu_{\mathrm{H}^{+}}^{g, \circ}-\Delta E_{\mathrm{ZP}}$ in Eqs. (1) and (2). $\Delta V$ stands for the HPS difference between the periodic interface and pure water models. (b) Schematic representation of the alignment of electrostatic potentials of bulk water in periodic interface and pure water models $\left(\phi_{\text {wat }}^{(i)}\right.$ and $\phi_{\text {wat }}^{(w)}$, respectively). 
TABLE I. Computed PZC vs SHE $\left(U_{\mathrm{PZC}}^{\circ}\right)$ and work functions $(\Phi)$ and decomposition of interface dipole potentials at the $\mathrm{Pt}(111), \mathrm{Au}(111), \mathrm{Pd}(111)$, and $\mathrm{Ag}(111)$ water interfaces using the PBE functional. The data in the parentheses are experimental values, taken from Refs [4,6,43-45] and references therein. $\Delta \Phi$ is the difference between the PZC and work function. $\Delta \Phi_{\mathrm{el}}$ and $\Delta \Phi_{\text {ori }}$ represent the changes of interfacial potentials due to electron redistribution and water orientation, respectively.

\begin{tabular}{lccccc}
\hline \hline Metal & $U_{\mathrm{PZC}}^{\circ}$ vs SHE $(\mathrm{V})$ & $\Phi(\mathrm{eV})$ & $\Delta \Phi(\mathrm{eV})$ & $\Delta \Phi_{\mathrm{el}}(\mathrm{eV})$ & $\Delta \Phi_{\text {ori }}(\mathrm{eV})$ \\
\hline $\mathrm{Pt}(111)$ & $0.2(0.3)$ & $5.8(5.9)$ & $-1.1(-1.2)$ & -1.3 & 0.2 \\
$\operatorname{Au}(111)$ & $0.5(0.5)$ & $5.4(5.4)$ & $-0.5(-0.5)$ & -0.5 & 0.0 \\
$\operatorname{Pd}(111)$ & $-0.5(0.1)$ & $5.1(5.6)$ & $-1.1(-1.0)$ & -1.0 & -0.1 \\
$\operatorname{Ag}(111)$ & $-0.6(-0.5)$ & $4.6(4.7)$ & $-0.8(-0.7)$ & -0.7 & -0.1 \\
\hline \hline
\end{tabular}

$$
e U_{\mathrm{PZC}}^{\circ}=-E_{\mathrm{F}}^{(i)}+\Delta_{\mathrm{DP}} A_{\mathrm{H}_{3} \mathrm{O}^{+}}^{(i)}-\mu_{\mathrm{H}^{+}}^{g, \circ}-\Delta E_{\mathrm{ZP}}
$$

in which $E_{F}^{(i)}$ is the Fermi energies of metal-water interfaces. Similarly, superscript (i) indicates both $E_{F}^{(i)}$ and $\Delta_{\mathrm{DP}} A_{\mathrm{H}_{3} \mathrm{O}^{+}}^{(i)}$ need to be computed in the same interface model. As illustrated in Fig. 1(a), the reference energy $\Delta_{\mathrm{DP}} A_{\mathrm{H}_{3} \mathrm{O}^{+}}^{(i)}$ differs from $\Delta_{\mathrm{DP}} A_{\mathrm{H}_{3} \mathrm{O}^{+}}^{(w)}$ in Eq. (1) for the water solution model. $\Delta_{\mathrm{DP}} A_{\mathrm{H}_{3} \mathrm{O}^{+}}^{(i)}$ is very expensive to calculate and has not yet be achieved for metal-water interface models.

To avoid the calculation of $\Delta_{\mathrm{DP}} A_{\mathrm{H}_{3} \mathrm{O}^{+}}^{(i)}$, we take an extra step by aligning the electrostatic potentials in bulk water,

$$
\Delta_{\mathrm{DP}} A_{\mathrm{H}_{3} \mathrm{O}^{+}}^{(w)}-\Delta_{\mathrm{DP}} A_{\mathrm{H}_{3} \mathrm{O}^{+}}^{(i)}=-e\left(\phi_{\mathrm{wat}}^{(w)}-\phi_{\mathrm{wat}}^{(i)}\right),
$$

in which $\phi_{\text {wat }}$ is the average electrostatic potential of the water phase, and the superscripts indicate the periodic models. Although both $\Delta_{\mathrm{DP}} A_{\mathrm{H}_{3} \mathrm{O}^{+}}$and $\phi_{\text {wat }}$ suffer from the reference uncertainty under PBCs, the HPS difference $(\Delta V)$ between $\Delta_{\mathrm{DP}} A_{\mathrm{H}_{3} \mathrm{O}^{+}}^{(w)}$ and $\Delta_{\mathrm{DP}} A_{\mathrm{H}_{3} \mathrm{O}^{+}}^{(i)}$ must be equal to that between $\phi_{\text {wat }}^{(w)}$ and $\phi_{\text {wat }}^{(i)}$. There is another implicit condition; in the Ewald sum the average electrostatic potential of a periodic box is set to zero, and thus $\phi_{\text {wat }}^{(w)}=0[21,41]$. Combining with Eqs. (2) and (3), we reach the central equation for computing the PZC vs SHE,

$$
e U_{\mathrm{PZC}}^{\circ}=-E_{F}^{(i)}-e \phi_{\mathrm{wat}}^{(i)}+\Delta_{\mathrm{DP}} A_{\mathrm{H}_{3} \mathrm{O}^{+}}^{(w)}-\mu_{\mathrm{H}^{+}}^{g, \circ}-\Delta E_{\mathrm{ZP}},
$$

in which both $E_{F}^{(i)}$ and $\phi_{\text {wat }}^{(i)}$ can be readily obtained by averaging over a $\sim 10$ ps DFTMD trajectory. This alignment procedure is illustrated in Fig. 1.

Born-Oppenheimer DFTMD simulations were carried out using the freely available CP2K/QUiCKSTEP package [42]. We used the Perdew-Burke-Ernzerhof (PBE) functional with Grimme's dispersion correction, and also hybrid HSE06 to check the functional dependence. The Pt(111), $\mathrm{Au}(111), \operatorname{Pd}(111)$ and $\mathrm{Ag}(111)$ surfaces were modeled by 3D periodic super cells consisting of a few layers of slabs and water regions [30].
The computed PZC of Pt(111), $\mathrm{Au}(111), \operatorname{Pd}(111)$ and $\operatorname{Ag}(111)$ water interfaces are listed in Table I, in comparison with the work functions $\Phi$ of the corresponding bare surfaces computed with a standard approach by referencing the Fermi energies with respect to the vacuum level. Our calculation can reproduce the experimental values within an uncertainty of $0.1 \mathrm{~V}$ [4,6,43-45]. An exception is $\operatorname{Pd}(111)$ that shows a large deviation of $0.6 \mathrm{~V}$. This can be attributed to the functional error in the Fermi energy of $\operatorname{Pd}(111)$ surface; Table I shows that there is a similar size of error in the $\Phi$ of $\operatorname{Pd}(111)$, in line with previous work [15], while the rest of the $\Phi$ are as accurate as the PZC predicted by the same PBE functional. This can be best seen from the difference $(\Delta \Phi)$ between PZC and $\Phi$, which can be regarded as a measure of interface dipole potentials formed by dipping metal surfaces into liquid water. It can be written as

$$
\Delta \Phi=\left(e U_{\mathrm{PZC}}^{\circ}+\Phi_{\mathrm{SHE}}\right)-\Phi,
$$

in which $U_{\mathrm{PZC}}^{\circ}$ vs SHE is converted into the absolute scale by adding the absolute SHE potential energy $\Phi_{\mathrm{SHE}}$. We use the value of $\Phi_{\mathrm{SHE}}=4.44 \mathrm{eV}$ recommended by Trasatti [18]. As shown in Table I, all $\Delta \Phi$ computed by the PBE functional are in good agreement with experiment including $\operatorname{Pd}(111)$. We have also calculated the $\operatorname{Pt}(111)$-water interface using hybrid HSE06, and found a consistent $\Delta \Phi$ (see Supplemental Material [30] for details).

Now, we turn to examine the magnitude of $\Delta \Phi$. The experimentally observed linear correlation between the PZC and $\Phi$ suggests a roughly constant $\Delta \Phi$ for $s p$ metals [3]. This is not consistent with our calculations on transition metals that show that there is a large variation in $\Delta \Phi$. Interface dipoles have been often attributed to two factors, namely, changes in water dipole orientation and metal surface potentials in contact with water [3].

We plot the profiles of oxygen density $\left(\rho_{\mathrm{H}_{2} \mathrm{O}}\right)$ and water orientation normal to the surfaces for $\mathrm{Pt}(111)$ and $\mathrm{Au}(111)$ in Fig. 2 [see Supplemental Material [30] for Pd(111) and $\mathrm{Ag}(111)]$. There are clear water adsorption peaks within $\sim 5 \AA$ from the surfaces, and the regions just above have a distinct lower density, separating surface water from the rest. Similar structures have been observed with classical MD [46] and quantum mechanics and molecular mechanics 


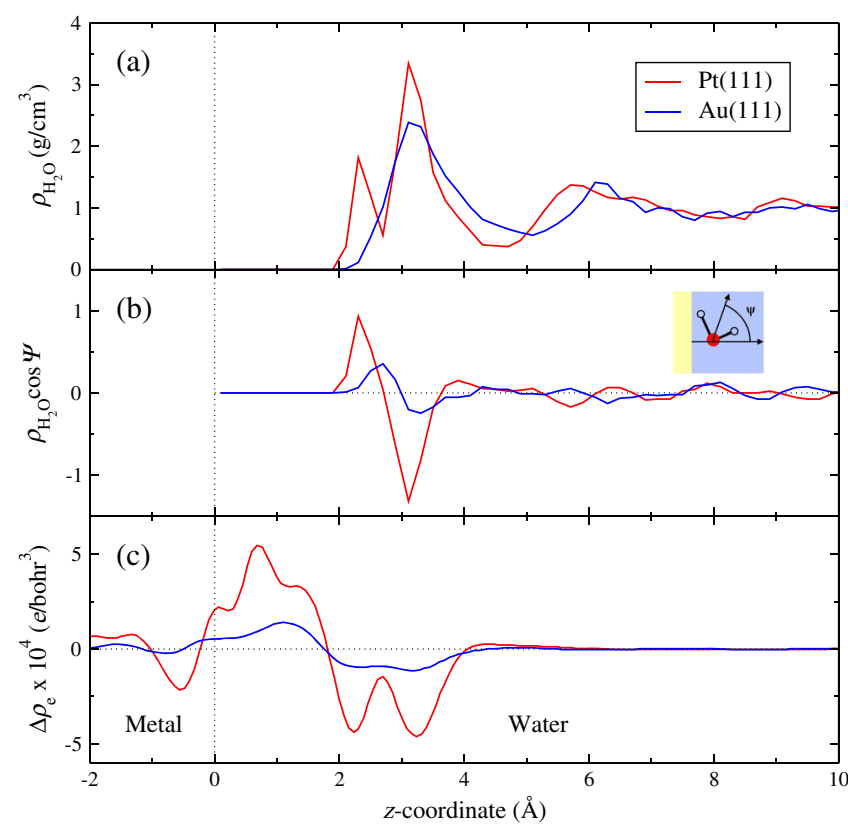

FIG. 2. The profiles of water density (a), dipole orientation (b), and electron redistribution (c) along the surface normal $z$ at the $\mathrm{Pt}(111)$ and $\mathrm{Au}(111)$ water interfaces. They are obtained by averaging over the two symmetric interfaces in our models. The zero in $z$ coordinates indicates the position of the metal surface.

$(\mathrm{QM}+\mathrm{MM})$ simulations [47], and explained by 2D hydrogen bonding networks within the surface water layers [46]. Water dipole orientation is expressed as $\rho_{\mathrm{H}_{2} \mathrm{O}} \cos \Psi$, where $\Psi$ is the angle between the water bisector and surface normal [see Fig. 2(b)], and it is weighted by water density, giving a direct measure of water dipole potentials. The water dipole orientation profile allows us to distinguish two types of water within the water adsorption peaks [see Fig. 3(a)]: (i) water adjacent to the metal surface with the dipole pointing away from the surface (i.e., upwards with positive $\cos \Psi$ ), denoted by watA; and (ii) water slightly further away from the surface with the dipole pointing downwards (negative $\cos \Psi)$, denoted by watB. Both are observed on all four metal surfaces, and the feature of watA is more pronounced on $\mathrm{Pt}$ and $\mathrm{Pd}$ than on $\mathrm{Au}$ and $\mathrm{Ag}$. Integrating $\rho_{\mathrm{H}_{2} \mathrm{O}} \cos \Psi$ leads to negligible net dipole orientation in the water adsorption layers on all four surfaces.

To investigate the electronic effects, we have calculated the charge redistribution profiles across the interfaces averaged over the DFTMD trajectories, $\Delta \rho_{e}(\mathrm{z})=\rho_{M / \mathrm{H}_{2} \mathrm{O}}(\mathrm{z})-$ $\rho_{M}(\mathrm{z})-\rho_{\mathrm{H}_{2} \mathrm{O}}(\mathrm{z})$, where $\rho_{M / \mathrm{H}_{2} \mathrm{O}}(\mathrm{z})$ is the electron density of the whole interface, and $\rho_{M}(\mathrm{z})$ and $\rho_{\mathrm{H}_{2} \mathrm{O}}(\mathrm{z})$ are the electron densities of the metal slab and water in separate at fixed geometries, respectively. As shown in Fig. 2(c), there is some electron accumulation (positive) between the surfaces and adsorbed water that mainly comes from the position above, and the magnitude of electron redistribution is much more pronounced on $\mathrm{Pt}(111)$ and on $\mathrm{Au}(111)$. This will give rise to a dipole effect and contribute to the interface dipole potential.

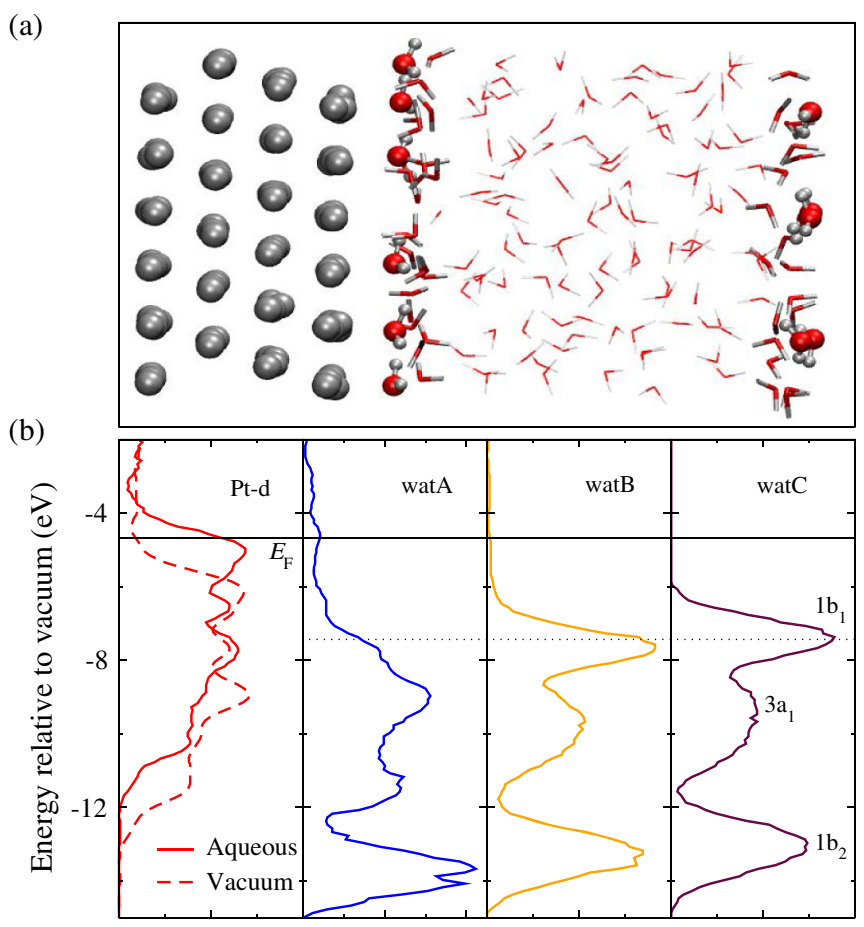

Density of states (arbi. units)

FIG. 3. (a) A snapshot of the $\mathrm{Pt}(111)$-water interface model. $\mathrm{Pt}, \mathrm{O}$, and $\mathrm{H}$ atoms are in silver, red, and white, respectively. Depending on the positions, wat $\mathrm{A}$, watB, and watC are distinguished and represented by balls, bonds, and lines, respectively. (b) Projected DOS plots of Pt and water at the interface computed by the PBE functional. They are averaged over a DFTMD trajectory.

Integrating the dipole along the surface normal, we can obtain the potential changes $\left(\Delta \Phi_{\mathrm{el}}\right)$ due to electron redistribution. The difference from $\Delta \Phi$ can be considered as the contribution of water orientation to the interface dipole potential, i.e., $\Delta \Phi_{\text {ori }}=\Delta \Phi-\Delta \Phi_{\mathrm{el}}$. It is clear from Table I that the electronic component $\Delta \Phi_{\mathrm{el}}$ dominates the interface dipole potential $\Delta \Phi$, and $\Delta \Phi_{\text {ori }}$ hardly contributes at the PZC conditions, confirming the structural analysis above that shows little net dipole orientation of surface water. It is worth noting that this is in opposition to common assumptions made in the analysis of electrochemical measurements of the PZC [5,6], and calls for reinterpretation of those results.

The electronic dipole potential $\Delta \Phi_{\mathrm{el}}$ is conventionally understood by electronic spillover using the jellium model, and contact with water results in a "pushback" effect of electron density and thus a decrease in work functions [3]. $A b$ initio calculation can offer detailed understanding of the electronic effects of the interface dipoles. We plot the density of states (DOS) near the interfaces, separating the contributions of water according to their distances from the surfaces (see Fig. 3(b) for Pt(111), and the others are given in Supplemental Material [30]). It can be found that the few water molecules directly adjacent to the surface (i.e., watA) chemically interact with $\mathrm{Pt}$, leading to rather distorted band 
states compared to the water molecules not significantly affected by the surface [watC, see Fig. 3(a)]. Mixing with metal $d$ bands, water $1 b_{1}$ and $3 a_{1}$ bands become broader and significantly downshift. In particular, the nonbonding $1 b_{1}$ states, due to the lone pair electrons of oxygen, make a major contribution to the bonding, which largely determines the watA geometry sitting rather flat with oxygen pointing to $\mathrm{Pt}$ and hydrogen slightly tilting up [48]. Furthermore, a fraction of water VB states penetrate above the Fermi level, indicating charge transfer from these water sates to Pt. This must be the cause of the large interface dipole potential. While the rest wat $\mathrm{B}$ in the adsorption layer only shows a slight change in the band position and shape. Similar to Pt, Pd also shows significant changes in the band structures of adsorbed water. As such, both have a large $\Delta \Phi$. Au and $\mathrm{Ag}$, however, form weaker bonding with water, and very few water VB states go beyond the Fermi levels. Thus, less charge is transferred, and smaller interface dipole potentials.

To summarize, we present a simple DFTMD method to compute the PZC of metal-water interfaces, which can be straightforwardly extended to calculate the band alignment of any aqueous interfaces. We also show that at the PZC conditions, the electronic effect owing to charge redistribution from surface water to the metal surfaces largely determines the interface dipole potentials; water orientation, however, has a negligible contribution.

J. Le thanks University of Aberdeen for a $\mathrm{PhD}$ studentship. We thank the United Kingdom Car-Parrinello (UKCP) consortium program on ARCHER, and Maxwell at University of Aberdeen for computing time. J. C. is grateful for funding support by the National Natural Science Foundation of China (Grants No. 21373166 and No. 21621091), and the Thousand Youth Talents Program of China.

*chengjun@xmu.edu.cn, jcheng@abdn.ac.uk

[1] M. Otani, I. Hamada, O. Sugino, Y. Morikawa, Y. Okamoto, and T. Ikeshoji, J. Phys. Soc. Jpn. 77, 024802 (2008).

[2] J. Rossmeisl, E. Skúlason, M. E. Björketun, V. Tripkovic, and J. K. Nørskov, Chem. Phys. Lett. 466, 68 (2008).

[3] W. Schmickler, Chem. Rev. 96, 3177 (1996).

[4] S. Trasatti and E. Lust, in Modern Aspects of Electrochemistry, edited by R.E. White (Kluwer Academic Publishers, Netherlands, 1999), pp. 1-198.

[5] T. Pajkossy and D. Kolb, Electrochim. Acta 46, 3063 (2001).

[6] A. Cuesta, Surf. Sci. 572, 11 (2004).

[7] R. Rizo, E. Sitta, E. Herrero, V. Climent, and J. M. Feliu, Electrochim. Acta 162, 138 (2015).

[8] W. Schmickler and R. Guidelli, Electrochim. Acta 127, 489 (2014).

[9] R. Jinnouchi and A. B. Anderson, Phys. Rev. B 77, 245417 (2008).

[10] H.-F. Wang and Z.-P. Liu, J. Phys. Chem. C 113, 17502 (2009).
[11] N. Bonnet and N. Marzari, Phys. Rev. Lett. 110, 086104 (2013).

[12] V. Tripkovic, M.E. Björketun, E. Skúlason, and J. Rossmeisl, Phys. Rev. B 84, 115452 (2011).

[13] S. Schnur and A. Groß, New J. Phys. 11, 125003 (2009).

[14] S. Sakong, K. Forster-Tonigold, and A. Groß, J. Chem. Phys. 144, 194701 (2016).

[15] L. S. Pedroza, A. Poissier, and M.-V. Fernández-Serra, J. Chem. Phys. 142, 034706 (2015).

[16] C. G. Van de Walle and R. M. Martin, Phys. Rev. B 35, 8154 (1987).

[17] T. A. Pham, C. Zhang, E. Schwegler, and G. Galli, Phys. Rev. B 89, 060202 (2014).

[18] S. Trasatti, Pure Appl. Chem. 58, 955 (1986).

[19] J. Cheng and M. Sprik, Phys. Rev. B 82, 081406 (2010).

[20] J. Cheng and M. Sprik, Phys. Chem. Chem. Phys. 14, 11245 (2012).

[21] J. Cheng, M. Sulpizi, and M. Sprik, J. Chem. Phys. 131, 154504 (2009).

[22] J. Cheng, X. Liu, J. VandeVondele, M. Sulpizi, and M. Sprik, Acc. Chem. Res. 47, 3522 (2014).

[23] J. Cheng and J. VandeVondele, Phys. Rev. Lett. 116, 086402 (2016).

[24] J. Cheng, X. Liu, J. A. Kattirtzi, J. VandeVondele, and M. Sprik, Angew. Chem., Int. Ed. Engl. 53, 12046 (2014).

[25] R. T. Tung, Appl. Phys. Rev. 1, 011304 (2014).

[26] V. Stevanović, S. Lany, D. S. Ginley, W. Tumas, and A. Zunger, Phys. Chem. Chem. Phys. 16, 3706 (2014).

[27] T. A. Pham, D. Lee, E. Schwegler, and G. Galli, J. Am. Chem. Soc. 136, 17071 (2014).

[28] N. Kharche, J. T. Muckerman, and M. S. Hybertsen, Phys. Rev. Lett. 113, 176802 (2014).

[29] F. Costanzo, M. Sulpizi, R. G. Della Valle, and M. Sprik, J. Chem. Phys. 134, 244508 (2011).

[30] See Supplemental Material at http://link.aps.org/ supplemental/10.1103/PhysRevLett.119.016801 for details of models and computational setup as well as validation and error analysis, which includes Refs. [31-40].

[31] J. P. Perdew, K. Burke, and M. Ernzerhof, Phys. Rev. Lett. 77, 3865 (1996).

[32] S. Grimme, J. Antony, S. Ehrlich, and H. Krieg, J. Chem. Phys. 132, 154104 (2010).

[33] S. Goedecker, M. Teter, and J. Hutter, Phys. Rev. B 54, 1703 (1996).

[34] C. Hartwigsen, S. Goedecker, and J. Hutter, Phys. Rev. B 58, 3641 (1998).

[35] J. VandeVondele and J. Hutter, J. Chem. Phys. 127, 114105 (2007).

[36] A. V. Krukau, O. A. Vydrov, A. F. Izmaylov, and G. E. Scuseria, J. Chem. Phys. 125, 224106 (2006).

[37] M. Guidon, J. Hutter, and J. VandeVondele, J. Chem. Theory Comput. 6, 2348 (2010).

[38] M. Guidon, J. Hutter, and J. VandeVondele, J. Chem. Theory Comput. 5, 3010 (2009).

[39] M. Guidon, F. Schiffmann, J. Hutter, and J. VandeVondele, J. Chem. Phys. 128, 214104 (2008).

[40] L. Schimka, J. Harl, A. Stroppa, A. GrÃneis, M. Marsman, F. Mittendorfer, and G. Kresse, Nat. Mater. 9, 741 (2010).

[41] S. W. de Leeuw, J.W. Perram, and E. R. Smith, Proc. R. Soc. A 373, 27 (1980). 
[42] J. VandeVondele, M. Krack, F. Mohamed, M. Parrinello, T. Chassaing, and J. Hutter, Comput. Phys. Commun. 167, 103 (2005).

[43] T. Pajkossy, T. Wandlowski, and D. M. Kolb, J. Electroanal. Chem. 414, 209 (1996).

[44] A. El-Aziz, L. Kibler, and D. Kolb, Electrochem. Comm. 4, 535 (2002).
[45] G. Valette, J. Electroanal. Chem. 269, 191 (1989).

[46] D. T. Limmer, A. P. Willard, P. Madden, and D. Chandler, Proc. Natl. Acad. Sci. U.S.A. 110, 4200 (2013).

[47] D. Golze, M. Iannuzzi, M.-t. Nguyen, D. Passerone, and J. Hutter, J. Chem. Theory Comput. 9, 5086 (2013).

[48] A. Michaelides, V. A. Ranea, P. L. de Andres, and D. A. King, Phys. Rev. Lett. 90, 216102 (2003). 